\title{
Solving the Structural Mysteries of Magnetic Materials in TEM
}

Y. Liu, CLAIM, Department of Mechanical Engineering

University of Michigan

2350 Hayward St.

Ann Arbor, MI 48109-2125

We are entering the era of optimum nanostructure design of magnetic materials for applications such as recording media, magnetic memory, magnetic sensors, exchange-coupled permanent magnets and spin electronics etc. Transmission electron microscopy (TEM) is the single powerful tool that can provide all the necessary information. This paper will review my recent experience in TEM of various magnetic materials. The first step is to use proper preparation method for different samples. Methods for bulk sample, thin films, self-assembled nanowires [1], and mechanically milled powders will be reviewed. The next step is to choose a proper technique to answer a particular question. Examples using selected area electron diffraction, convergent beam electron diffraction, nanodiffraction, high resolution TEM [2], super-resolution TEM, selected reflection imaging (SRI) [3], compositional mapping by electron energy loss spectroscopy (EELS) [4] and by energy dispersive X-ray spectroscopy, and Lorentz microscopy will be shown [5].

Figure 1 is nanodiffraction patterns used to measure the volume fraction of amorphous phase and to identify the crystal structure of the crystallite phase in Co-Sm film. It is found that higher Ar pressure during deposition and higher concentration of Sm promote the formation of amorphous phase. This method is most accurate without limitation of the resolution of the TEM. Using nanodiffraction several stacking modes such as $\mathrm{AB}, \mathrm{ABC}$, and other modes were identified in CoSm films. Figure 2 (a) is diffraction pattern, (b) is (002) SRI image showing the grains with the preferred direction and (c) is (001) SRI image of FePt:Fe composite film. The bright grains in Figure 2 (c) is ordered $\mathrm{L} 1_{0}$ phase while those that are white in Figure 2 (b) but dark in Figure 13 (c) are Fe solid solution. This method is most sensitive to the ordering regardless of the chemical composition of the phases. The identification of the Fe phase and FePt phase is critical for explanation of the exchange coupling in this composite system. Figure 3 is the HRTEM image of $\mathrm{Sm}_{2}(\mathrm{CoFeCuZr})_{17}$ system high temperature permanent magnet. $\mathrm{Zr}$ exists in the precipitate with a coherent lattice with the matrix. Such structure is stable at high temperature and the Zr-rich precipitates enhance the coercivity by pinning the domain wall movement.

\section{References}

[1] Y. Liu, M. Zhen, H.Zeng and D. J. Sellmyer, Nanophase and Nanostructured Materials, Eds. Z. L. Wang, Y. Liu and Z. Zhang, Tsinghua Univresity Press and Kluwer Accademic/ Plenum Publishers, Vol. 3, (2001) 210.

[2] Y. Liu, D.J. Sellmyer, B. W. Rob ertson, Z. S. Shan, and S. H. Liou, IEEE Trans. on Magn., Vol. 31, (1995) 2740.

[3] Y. Liu and D. J. Sellmyer. Proc. of Microscopy and Microanalysis 1998, Atlanta, Georgia, 752.

[4] Y. Liu, C. Nelson etc. Proc. of Microscopy and Microanalysis 2002.

[5] Y. Liu, Z. S. Shan, and D. J. Sellmyer, IEEE Trans. on Magn., Vol. 32,(1996) 3614.

[6] Most of this work was performed at CMRA, University of Nebraska at Lincoln and supported by CMRA, NSIC, DOE and NSF. 

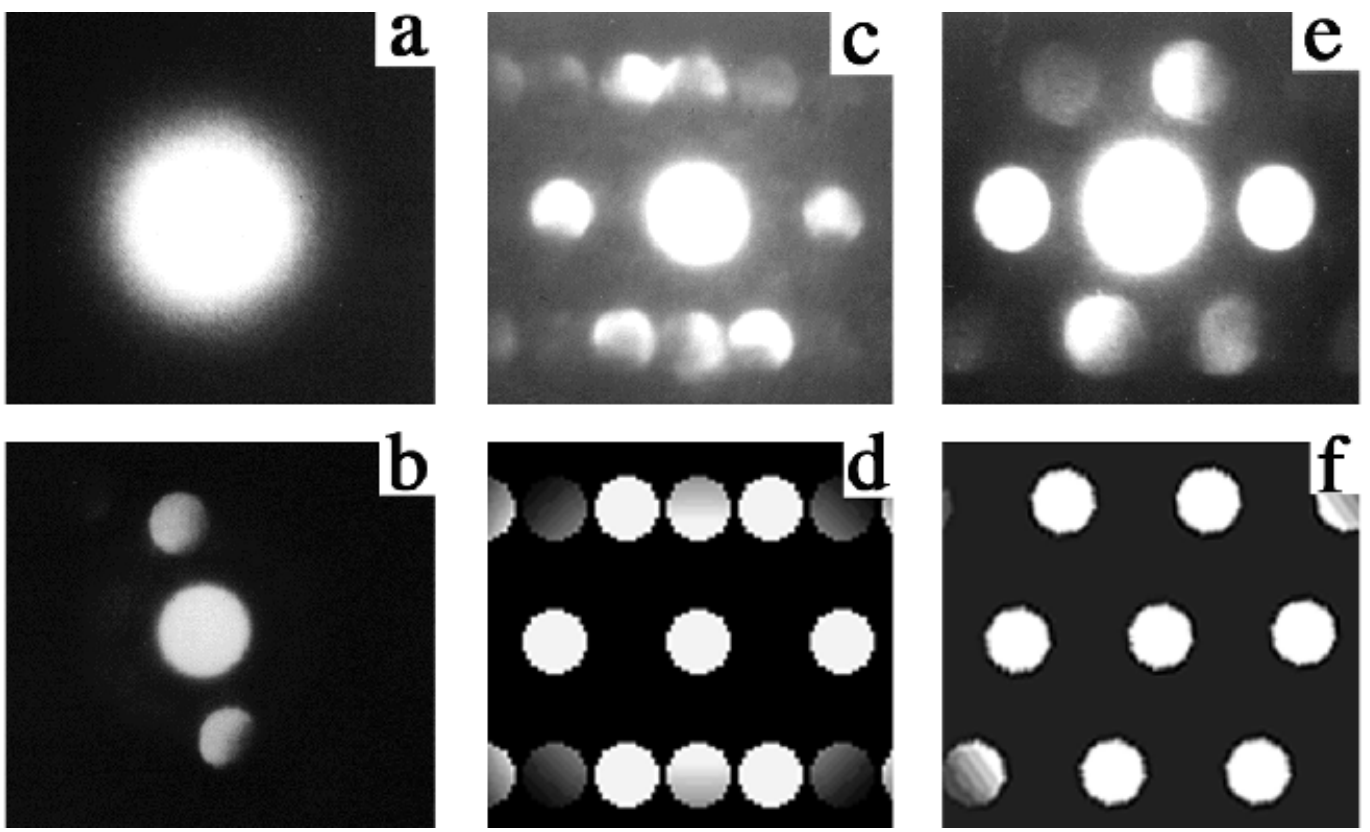

FIG. 1. Nano-diffraction patterns: (a) from amorphous phase, (b) from a crystallite phase, (c) is TEM pattern, (d) is simulated [11.0] zone axis pattern of HCP structure, (e) is TEM pattern, and (f) is simulated [110] zone axis pattern of FCC structure. Several stacking modes have been identified in the as-deposited Co-Sm films.

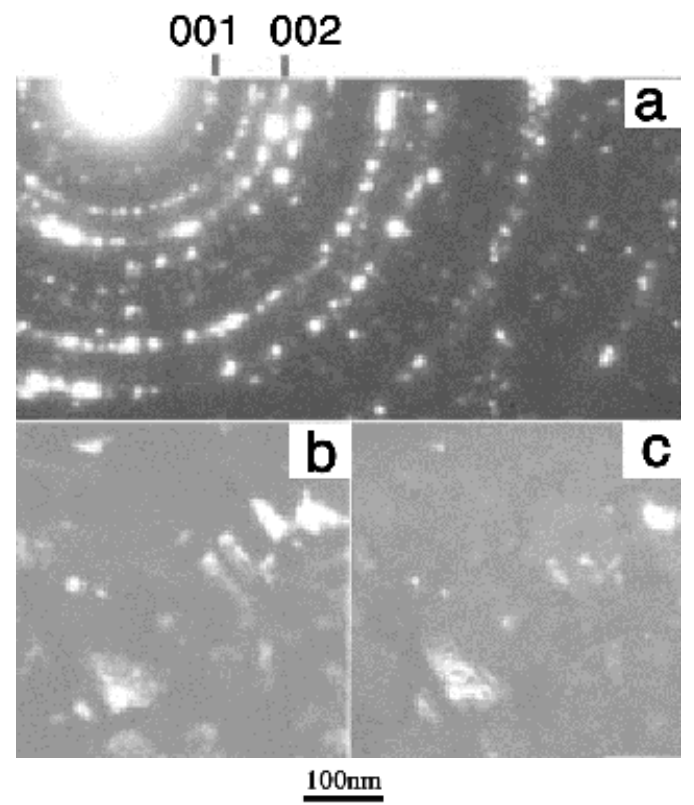

FIG. 2. (a) diffraction pattern (b) is the (002) SRI image (c) is (001) reflection image of FePt:Fe composite film.

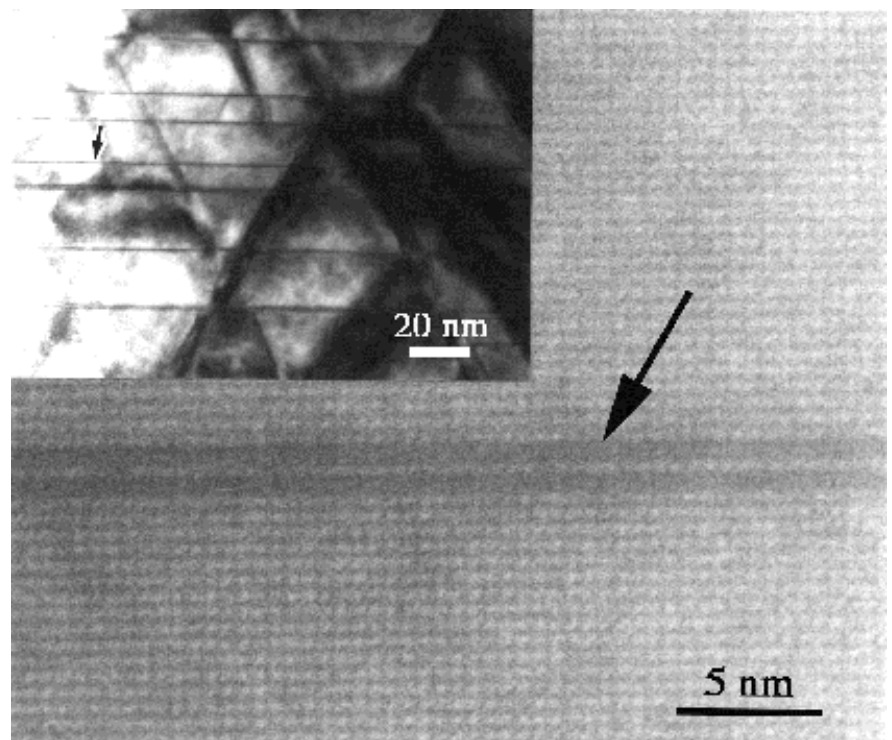

FIG. 3. Bright field and HRTEM image of a $\mathrm{Sm}_{2}(\mathrm{CoFeCuZr})_{17}$ magnet. 